\title{
Locality and Scaling of Quenched Overlap Fermions $*$
}

$\chi$ QCD Collaboration: Terrence Draper ${ }^{\dagger a}$, Nilmani Mathur ${ }^{a, b}$, Jianbo Zhang ${ }^{c}$, Andrei Alexandru $^{a}$, Ying Chen ${ }^{d}$, Shao-Jing Dong ${ }^{a}$, Ivan Horváth ${ }^{a}$, Frank Lee ${ }^{e}$, and Sonali Tamhankar ${ }^{a, f}$

${ }^{a}$ Department of Physics and Astronomy, University of Kentucky, Lexington, KY 40506, USA

${ }^{b}$ Jefferson Lab, 12000 Jefferson Avenue, Newport News, VA 23606, USA

${ }^{c}$ CSSM and Department of Physics, University of Adelaide, Adelaide, SA 5005, Australia

${ }^{d}$ Institute of High Energy Physics, Academia Sinica, Beijing 100039, P.R. China

${ }^{e}$ Center for Nuclear Studies, Dept. of Physics, George Washington Univ., Washington, DC 20052, USA

${ }^{f}$ Department of Physics, Hamline University, St. Paul, MN 55104, USA

E-mail: draper@pa.uky.edu

The overlap fermion offers the tremendous advantage of exact chiral symmetry on the lattice, but is numerically intensive. This can be made affordable while still providing large lattice volumes, by using coarse lattice spacing, given that good scaling and localization properties are established. Here, using overlap fermions on quenched Iwasaki gauge configurations, we demonstrate directly that the overlap Dirac operator's range is comfortably small in lattice units for each of the lattice spacings $0.20 \mathrm{fm}, 0.17 \mathrm{fm}$, and $0.13 \mathrm{fm}$ (and scales to zero in physical units in the continuum limit). In particular, our direct results contradict recent speculation that an inverse lattice spacing of $1 \mathrm{GeV}$ is too low to have satisfactory localization. Furthermore, hadronic masses (available on the two coarser lattices) scale very well.

XXIIIrd International Symposium on Lattice Field Theory

25-30 July 2005

Trinity College, Dublin, Ireland

${ }^{*}$ This research is supported in part by the U.S. Department of Energy under grants DE-FG05-84ER40154, DEFG02-02ER45967, and DE-FG02-95ER40907.

† Speaker. 


\section{Locality}

In the last several years the use of the overlap fermion has become more popular because the conceptual and technical clarity that results from its exact chiral symmetry on the lattice is seen to overcome its superficially higher computational cost as compared to conventional formulations of the fermion. Furthermore, the disparity in numerical intensity can be mitigated by using coarse lattice spacing, given that good scaling and localization properties are established.

Hernández, Jansen, and Lüscher [1] showed numerically that Neuberger's overlap operator is local (using the Wilson gauge action on fine lattices). Recently, Golterman, Shamir, and Svetitsky [2] have speculated that overlap simulations with a cutoff of $1 \mathrm{GeV}$ (such as [3]) might have a range as long as 4 lattice units, and thus be afflicted by unphysical degrees of freedom as light as $0.25 \mathrm{GeV}$. Here we show directly that such is not the case; the range is about 1 lattice unit (in Euclidean distance or 2 units of "taxi-driver" distance). All is well.

\subsection{Lattice Details}

We use the renormalization-group-improved Iwasaki [4] gauge action, on three different lattices; for each, the lattice size, lattice spacing, and number of configurations used are tabulated in Table 1.

\begin{tabular}{llr}
$N_{s} \times N_{t}$ & $a(\mathrm{fm})$ & $N_{\mathrm{cfg}}$ \\
\hline $16^{3} \times 28$ & 0.20 & $300 / 10$ \\
$20^{3} \times 32$ & 0.17 & $98 / 10$ \\
$28^{3} \times 44$ & 0.13 & $/ 10$ \\
\hline
\end{tabular}

Table 1: Lattice size, lattice spacing, number of configurations (for scaling/locality).

For the associated scaling study of hadron masses, we use the overlap fermion [5] and massive overlap operator [6]

$$
D\left(m_{0}\right)=\left(\rho+\frac{m_{0} a}{2}\right)+\left(\rho-\frac{m_{0} a}{2}\right) \gamma_{5} \varepsilon(H)
$$

where $\varepsilon(H)=H / \sqrt{H^{2}}, H=\gamma_{5} D_{w}$, and $D_{w}$ is the usual Wilson fermion operator, except with a negative mass parameter $-\rho=1 / 2 \kappa-4$ in which $\kappa_{c}<\kappa<0.25$; we take $\kappa=0.19$ in our calculation which corresponds to $\rho=1.368$. For the locality study, we set $m_{0}=0$ to look at the properties of the massless operator $D(0)$. Complete details are described in [3].

\subsection{Locality as Measured by Taxi-Driver Distance}

It is convenient for formal reasons to discuss locality in terms of "taxi-driver" distance [1].

$$
r_{\mathrm{TD}}=\|x-y\|_{1}=\sum_{\mu}\left|x_{\mu}-y_{\mu}\right|
$$

The locality of the overlap operator is then studied by plotting the quantity $|D(r)|(f(r)$ in the notation of [1]) as a function of the taxi-driver distance for a localized source, $\psi_{\alpha}(x)=\delta(x) \delta_{\alpha \beta}$ 
for fixed Dirac-color index $\beta$.

$$
|D(r)|=\max \left\{|| D \psi(x)|| \mid \sum_{\mu} x_{\mu}=r\right\}
$$

For large $r$, the kernel of the Dirac-overlap operator decays exponentially with decay rate $v=r_{\mathrm{ov}}^{-1}$, where $r_{\mathrm{ov}}$ is the range (characteristic decay distance) measured in lattice units.

\subsection{Results}

In the left pane of Fig. 1, we plot $|D(r)|$ as a function of taxi-driver distance for each of three lattice spacings. At large distances, we fit to an exponentially decreasing function to extract the range $r_{\mathrm{ov}}$. These are tabulated in Table 2. We note that our results are consistent with the results of Hernández, Jansen and Lüscher [1] on finer lattices for the overlap operator $(\rho=1.4)$ with Wilson action at $\beta=6.0,6.2$, and 6.4 where they find $v=r_{\mathrm{ov}}^{-1}=0.49$.

\begin{tabular}{ll}
$a$ & $r_{\mathrm{ov}}$ \\
\hline $0.20 \mathrm{fm}$ & $1.93(1)$ \\
$0.17 \mathrm{fm}$ & $1.83(1)$ \\
$0.13 \mathrm{fm}$ & $1.81(1)$ \\
\hline
\end{tabular}

Table 2: The range (taxi driver metric) for three lattice spacings. It is less than two lattice units on a lattice as coarse as $0.20 \mathrm{fm}$.
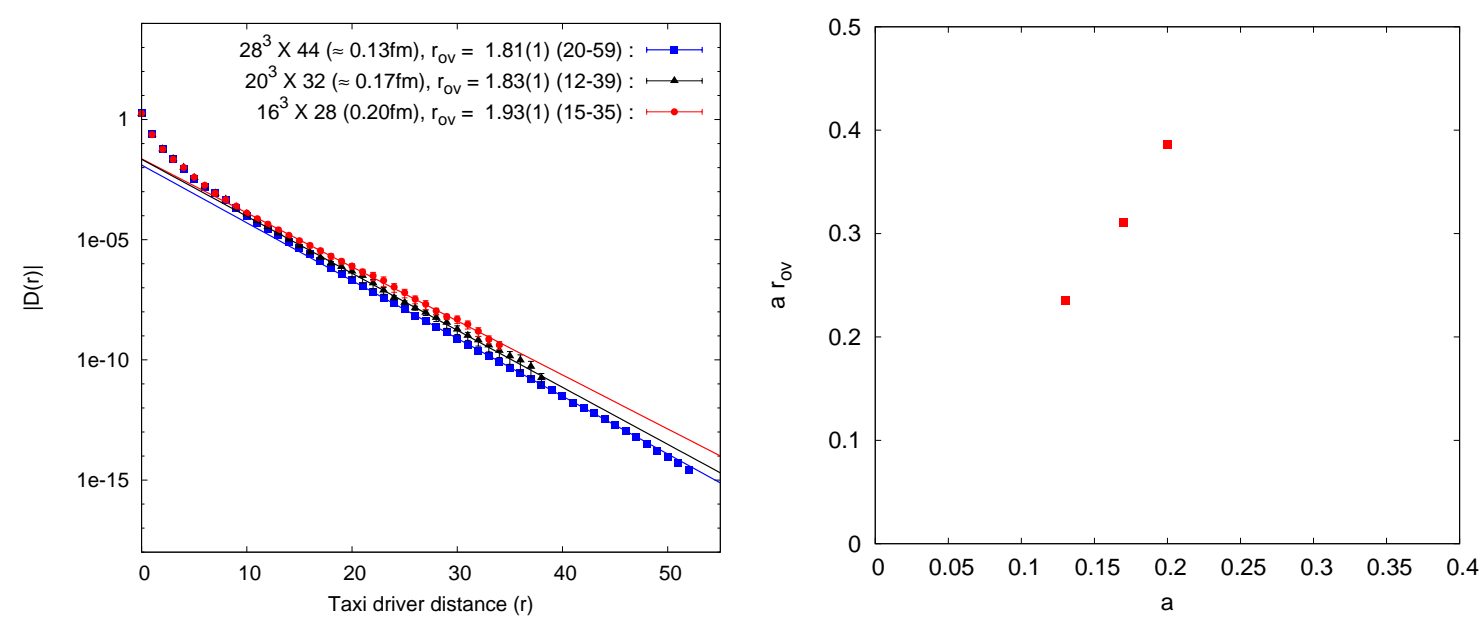

Figure 1: Left: For each of three lattice spacings, the expectation value of $|D(r)|$ as a function of the taxi-driver distance, $r$. For large $r, \mid D(r)) \mid$ falls exponentially, with range $r_{\mathrm{ov}}$. Fitted values of $r_{\mathrm{ov}}$ are shown with fit intervals. Right: Taxi driver range in physical units ( $\mathrm{fm}$ ) as a function of lattice spacing. The range is small even at coarse lattice spacing and trends to zero in the continuum limit.

Furthermore, it is gratifying to see that even for our coarsest lattice, $0.20 \mathrm{fm}$, the measured range is less than two lattice units. In the right pane of Fig. 1 we plot the range in physical units as a function of lattice spacing; it trends to zero in the continuum limit. 


\subsection{Locality as Measured by Euclidean Distance}

We conclude that it is perfectly acceptable to simulate overlap fermions with lattice spacing as coarse as $0.20 \mathrm{fm}$, since for this we find that the range is not greater than two lattice units when measured in taxi-driver distance. In fact, the situation is even better than it seems. To see this, we consider the more familiar standard Euclidean metric

$$
r_{\mathrm{E}}=\|x-y\|_{2}=\sqrt{\sum_{\mu}\left|x_{\mu}-y_{\mu}\right|^{2}}
$$

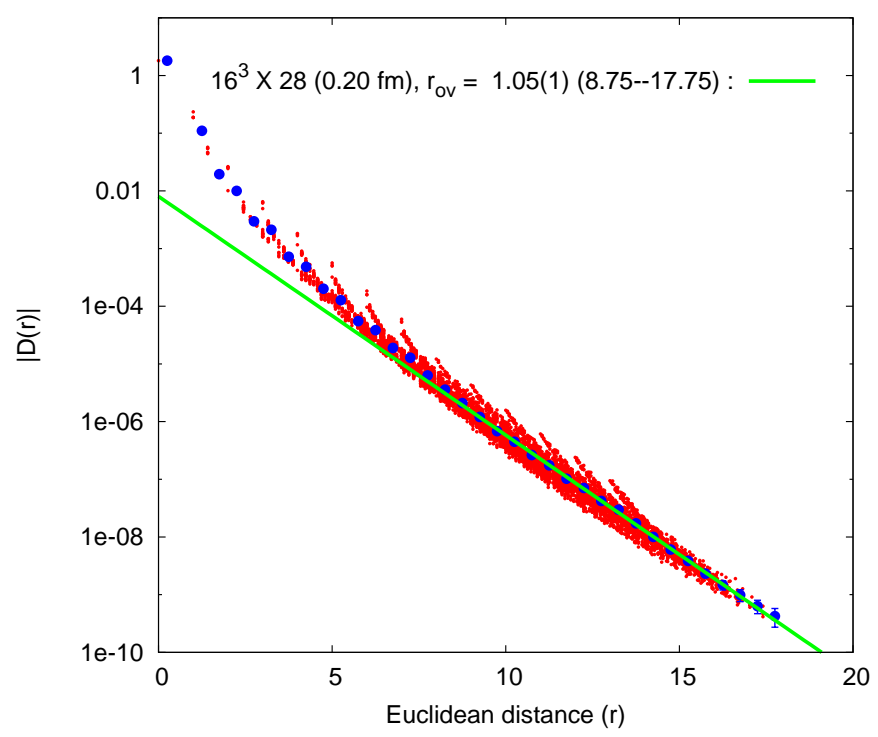

Figure 2: For the $16^{3} \times 28$ lattice, the expectation value of $|D(r)|$ as a function of Euclidean distance $r$ (for data cut to remove wrap-around effects). Data is plotted at each Euclidean distance in red. The blue points are averages within bins of width $\Delta r=0.5$. The exponential tail is then fit over the longest interval possible with acceptable $\chi^{2}$, as shown by the green line which has inverse slope $r_{\mathrm{ov}}=1.05(1)$, the range.

In Fig. 2 we plot $D(r)$ versus the Euclidean distance for our coarsest lattice. As compared to the corresponding plot 1 using the less-familiar taxi-driver distance, one sees that the data are more scattered due to violations of rotational symmetry, but are still clearly contained with a worst-case decay rate.

\begin{tabular}{ll}
$a$ & $r_{\mathrm{ov}}$ \\
\hline $0.20 \mathrm{fm}$ & $1.05(1)$ \\
$0.17 \mathrm{fm}$ & $0.98(1)$ \\
$0.13 \mathrm{fm}$ & $0.9(1)$ \\
\hline
\end{tabular}

Table 3: The range (Euclidean metric) at three values of lattice spacing. It is less than about 1 lattice unit with lattice spacing as coarse as $0.20 \mathrm{fm}$. 
Again we fit the tail of the function with a decaying exponential to extract the range. We tabulate the results in Table 3. Note that although the two ranges (taxi-driver and Euclidean) differ by a factor of two they are quite compatible heuristically; on a $L^{4}$ hypercube, the maximum taxidriver distance is $4 L$, and the maximum Euclidean distance is $\sqrt{4 L^{2}}=2 L$.

So even at lattices as coarse as $a=0.2 \mathrm{fm}$, the range is about 1 lattice unit (measured using Euclidean distance, or 2 units using taxi-driver distance). No unphysical degrees of freedom are induced at distances longer than the lattice cutoff.

\section{Scaling}

At Lattice 2004, Davies et al. [7] collected world data to demonstrate that different quenched quark formulations could have a consistent continuum limit.

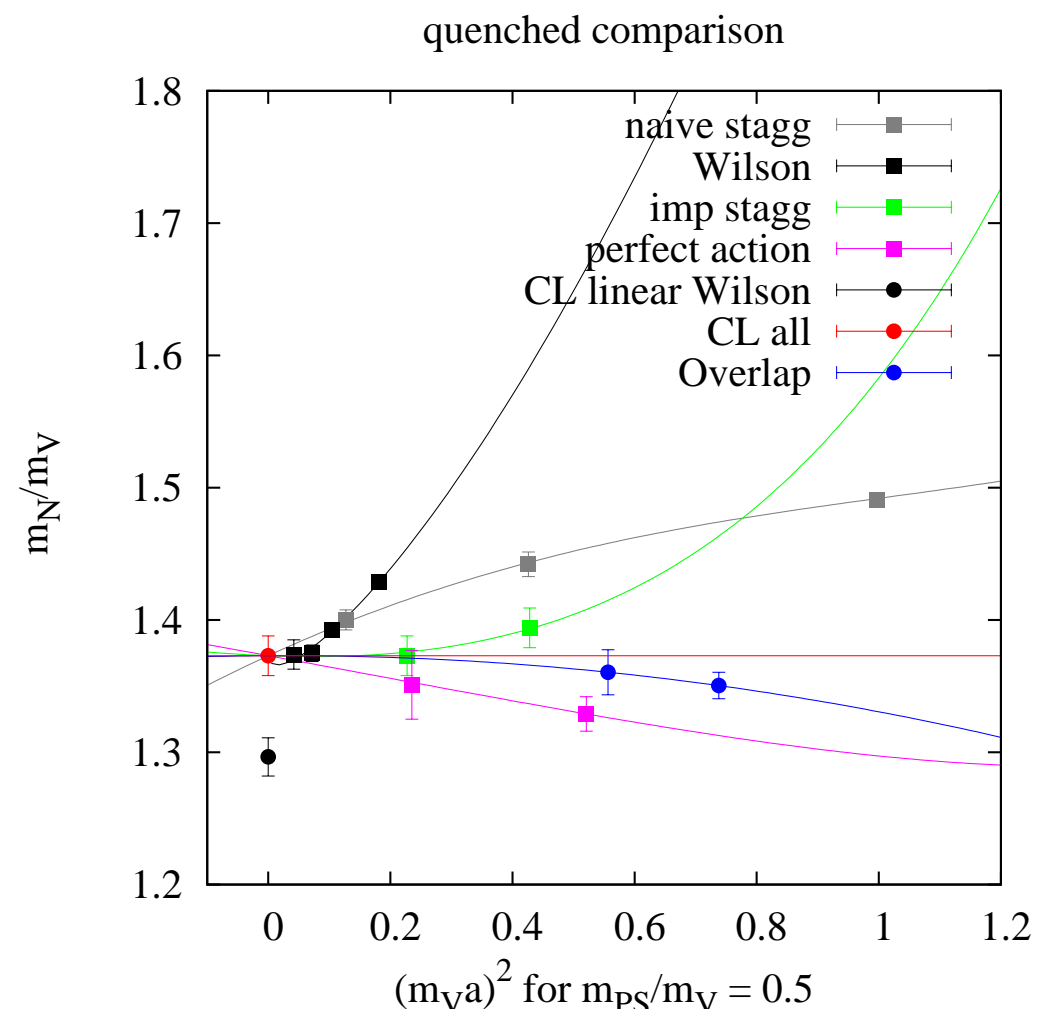

Figure 3: "Aoki" plot for various quenched data, as obtained from [7]. Our data is labeled "overlap".

The conclusion, as illustrated in Fig. 3, is that they could. But we emphasize that the constrained fit demanded that there exist a global continuum limit (by design). Furthermore, the global continuum limit differed substantially from the continuum limit obtained solely from the Wilson formulation where the discretization errors are largest. The lesson learned is that with large discretization errors, it is quite possible to be misled when extrapolating to the continuum limit, even with high statistics and many lattice spacings. It is important to seek a formulation with very small discretization errors to be able to trust the continuum extrapolation. 
Here we add our data "overlap" to the global quenched spectrum data and find that of all the formulations, its discretization errors are smallest, allowing for viable computation at surprisingly coarse lattice spacing.

As another example of the efficacy of the overlap formulation, we made a non-perturbative computation [8] of the renormalization constants of composite operators on the $16^{3} \times 28$ lattice using the regularization independent scheme.

We found that the relations $Z_{A}=Z_{V}$ and $Z_{S}=Z_{P}$ agree well (within $1 \%$ ) above $m=1.6 \mathrm{GeV}$. The $m_{\Lambda_{\mathrm{QCD}}} a^{2}$ and $(m a)^{2}$ corrections of the renormalization are small; the mass dependence is less than about $3 \%$ up to $m a=0.6$. This is much superior to the competition.

\section{Conclusions}

It is viable to simulate quenched overlap fermions at surprisingly coarse lattice spacing. Locality is well under control; the range (characteristic exponential decay length) is about one lattice unit (of Euclidean distance, or about two lattice units of Taxi-driver distance) for lattice spacing as coarse as $0.20 \mathrm{fm}$ (such as in [3]), and trends to zero (in physical units) in the continuum limit. Scaling is remarkable. The Aoki plot is essentially flat up to $0.20 \mathrm{fm}$. The overlap fermion outperforms all other formulations; discretization errors are smallest for overlap. Non-perturbative renormalization of operators show little mass dependence [8]; e.g. less than about $3 \%$ up to $m a=0.6$ for the renormalization constants

\section{References}

[1] P. Hernández, K. Jansen and M. Lüscher, Locality properties of Neuberger's lattice Dirac operator, Nucl. Phys. B 552, 363 (1999).

[2] M. Golterman, Y. Shamir and B. Svetitsky, Localization properties of lattice fermions with plaquette and improved gauge actions, arXiv:hep-lat/0503037.

[3] Y. Chen at al., Chiral Logs in Quenched QCD, Phys. Rev. D70, 034502 (2004).

[4] Y. Iwasaki, Renormalization group analysis of lattice theories and improved lattice action, Nucl. Phys. B 258, 141 (1985).

[5] H. Neuberger, Exactly massless quarks on the lattice, Phys. Lett. B417, 141 (1998); R. Narayanan and H. Neuberger, A construction of lattice chiral gauge theories, Nucl. Phys. B443, 305 (1995).

[6] C. Alexandrou et al., One-loop renormalization of fermionic currents with the overlap-Dirac operator, Nucl. Phys. B580, 394 (2000); S. Capitani, Perturbative renormalization of moments of quark momentum, helicity and transversity distributions with overlap and Wilson fermions, Nucl. Phys. B592, 18 (2001); P. Hernández et al., Non-perturbative renormalization of the quark condensate in Ginsparg-Wilson regularizations, JHEP 0107, 018 (2001).

[7] C.T.H. Davies et al., The quenched continuum limit, Nucl. Phys. Proc. Suppl. 140, 261 (2005), [arXiv:hep-lat/0409039], and references therein.

[8] J.B. Zhang et al., Nonperturbative renormalization of composite operators with overlap fermions, arXiv:hep-lat/0507022. 